\title{
Design of Korean Noninvasive Risk Evaluation Study for Sudden Cardiac Death from Infarction or Heart Failure - Myocardial infarction study of K-REDEFINE registry -
}

Seung-Jung Park, MD1; Gyo-Seung Hwang, MD2; Gi-Byoung Nam, MD³; Hyung Wook Park, MD4; Joong-Wha Chung, $\mathrm{MD}^{5}$; Seung Yong Shin, MD'; Sang Min Kim, MD7; Jun Hyung Kim, MD ${ }^{8}$; Young Soo Lee, MD $^{9}$; Yae Min Park, MD10; Jong-Youn Kim, MD11; Dae Hyeok Kim, MD12; Dae kyeong Kim, MD13; June Namgung, MD14; Dae-Hee Shin, MD ${ }^{15}$; Joon Hyouk Choi, MD'16; Hyoung-Seob Park, MD17; Jong-Il Choi, MD ${ }^{18}$; Jin-Seok Kim, MD ${ }^{19}$; Tae Joon Cha, MD20; Sang Weon Park, MD21; Jae-Sun Uhm, MD22; Nam-Ho Kim, MD23; Minsoo Ahn, MD24; Dong-Gu Shin, MD25; Nuri Jang ${ }^{1}$; Meemo Park'1; June Soo Kim, MD1; on behalf of the K-REDEFINE investigators

\footnotetext{
ISamsung Medical Center, Sungkyunkwan University School of Medicinc, Scoul, Republic of Korea; ${ }^{2}$ Ajou University Hospital, Suwon, Republic of Korea; ${ }^{3}$ Asan Medical Center, Scoul, Republic of Korea; ${ }^{4}$ Chonnam National University Hospital, Gwangju, Republic of Korea; ${ }^{5}$ Chosun university hospital, Gwangju, Republic of Korea; ${ }^{6}$ Chung-Ang University Hospital, Scoul, Republic of Korea: ${ }^{7}$ Chungbuk National University Hospital, Cheongju, Republic of Korea; ${ }^{8}$ Chungnam National University Hospital, Dacjeon, Republic of Korea; ${ }^{9}$ Dacgu Catholic University Medical Center, Daegu, Republic of Korea; ${ }^{10}$ Gachon University Gil Medical Center Incheon, Republic of Korea; " Republic of Korea; ${ }^{12}$ Inha university hospital, Incheon, Republic of Korea; ${ }^{13}$ Inje University Busan Paik Hospital, Busan, Republic of Korea; ${ }^{14}$ Inje University llsan Paik Hospital, Goyang, Republic of Korea; ${ }^{5}$ International St. Mary's Hospital, Incheon, Republic of Korea; ${ }^{16}$ jeju National University Hospital, Jeju, Republic of Korea; ${ }^{17}$ Keimyung university dongsan medica center, Dacgu, Republic of Korea; ${ }^{18}$ Korea University Medical Center, Seoul, Republic of Korea; ${ }^{19}$ Korea University Ansan Hospital, Ansan, Republic of Korea; ${ }^{20}$ Kosin University Gospel Hospital, Busan, Republic of Korea; ${ }^{21}$ Sejong General Hospital, Bucheon, Republic of Korea; ${ }^{22}$ Severance Hospital, Yonsei University College of Medicine, Seoul, Republic of Korea ${ }^{23}$ Wonkwang University Hospital, Iksan, Republic of Korea; ${ }^{24}$ Wonju Severance Christian Hospital, Wonju, Republic of Korea; ${ }^{25}$ Yeungnam University Hospital, Dacgu, Republic of Korea
}

Received: October 19, 2016

Revision Received: November 29, 2016

Accepted: December 6, 2016

Correspondence: June Soo Kim, MD, PhD

Division of Cardiology, Department of Medicine,

Cardiac \& Vascular Center, Samsung Medical

Center, Sungkyunkwan University School of

Medicine, 81, Irwon-ro Gangnam-gu, Seoul

06351, Republic of Korea

Tel: +82-2-3410-3414, Fax: +82-2-3410-3417

Email: juneskim@skku.edu, js58.kim@samsung.com

Copyright (C) 2017 The Official Journal of Korean Heart Rhythm Society Editorial Board and MMK

Communications Limited

\section{ABSTRACT}

Background and Objectives: Despite significant advances in the treatment of acute myocardial infarction (MI), the prevention of sudden cardiac death (SCD), the most common mode of death in patients with $\mathrm{MI}$, remains challenging. Furthermore, previous Korean MI registries did not address the issue of post-MI SCD. Additional risk stratifiers of post-MI SCD are still required to compensate for the limitation of using left ventricular ejection fraction to predict lethal arrhythmic events.

Subjects and Methods: We designed the first Korean prospective nationwide multicenter registry primarily focused on SCD; the Korean noninvasive Risk Evaluation study for sudden cardiac DEath From INfarction or heart failurE (K-REDEFINE). The registry consists of 2 groups of patients presenting with (1) acute $\mathrm{MI}$ or (2) acute heart failure (HF) at 25 tertiary referral cardiovascular centers. The primary endpoint of the $\mathrm{MI}$ group study of K-REDEFINE registry is the incidence and risk factors of post-MI SCD. In particular, the association between the risk of SCD and non-invasive Holter-based electrocardiogram (ECG) variables will be evaluated, such as T-wave alternans (marker of repolarization heterogeneity) and heart rate turbulence/variability (a marker of autonomic function). Other secondary study outcomes include atrioventricular arrhythmias, HF-related admission, repeated myocardial ischemic events, stroke, and overall deaths.

Conclusion and Perspective: The K-REDEFINE registry will provide new prospects for the better management of MI patients with high risk of SCD by clarifying the burden and predictors of SCD and the clinical utility of various non-invasive ambulatory ECG-based variables in risk stratification for SCD in this patient population.

Key Words: - Sudden Cardiac Death -Myocardial Infarction -Electrocardiography -Ambulatory 


\section{Introduction}

Despite recent significant advances in the treatment of acute myocardial infarction (MI), sudden cardiac death (SCD) continues to be the most common cause of death after acute MI, acomprising $30-40 \%$ of overall mortality according to Western studies. ${ }^{1,2}$ The incidence of acute MI in Korea has also increased by around $10 \%$ each year during the last several decades, ${ }^{3}$ with the 1 -year mortality (11\%) comparable to those from many developed countries due to westernization of lifestyles and a rapidly aging population. ${ }^{4}$ Moreover, with the advent of potentially life-saving therapy such as implantable cardioverterdefibrillator (ICD), a more accurate risk stratification of patients post-MI becomes more critical in clinical practice.

Left ventricular (LV) ejection fraction (EF) has been one of the most widely used criteria for determining ICD implantation as a primary prevention strategy against post-MI SCD..$^{5-7}$ However, more than half of SCD cases developed in MI survivors with a LV $\mathrm{EF}>40 \%$, showing a low positive predictive value of LV EF. ${ }^{8}$ Additionally, in a recent study that enrolled post-MI patients with a reduced $\operatorname{LV}$ EF ( $\leq 40 \%)$, lethal ventricular tachyarrhythmia was documented in only $8.0 \%$ over a 2 -year follow-up period. ${ }^{9}$ Prophylactic ICD use after acute MI failed to show mortality benefit when used in the first month during which the risk of SCD was reportedly highest. ${ }^{10}$

Given the limitation of LV EF as a risk factor, continuous efforts to find another risk factor of SCD in patients with acute MI are required. ${ }^{11}$ T-wave alternans (TWA) and heart rate turbulence (HRT) are non-invasive surrogate markers of cardiac electrical instability and autonomic dysfunction, respectively. A close association between the abnormal values of TWA and/or HRT and an increased risk of SCD has been suggested by many clinical studies. ${ }^{12-15}$ However, the predictive values of TWA and HRT for SCD have never been evaluated in Korean patients following acute MI.

Therefore, we aimed to assess the incidence and risk factors of SCD in patients with acute MI using a newly designed nationwide multicenter MI registry, and evaluate the efficacy of TWA and HRT alone or in combination with other parameters for predicting SCD in this patient population.

\section{Subjects and Methods}

\author{
Study population
}

The K-REDEFINE (Korean noninvasive Risk Evaluation study for sudden cardiac DEath From INfarction or heart failure) is designed as a prospective nationwide multicenter registry encompassing 2 groups of patients presenting with (1) acute MI or (2) acute HF at 25 tertiary referral cardiovascular centers in South Korea. Enrollment of patients began in September 2015 and is expected to be completed by the first half of 2018. Followup is planned for up to 5 years after finishing the enrollment.

Patients who show evidence of myocardial necrosis as suggested by a rise and/or fall of cardiac enzyme (preferably troponin) with at least one of the following will be screened for enrollment eligibility into the acute MI study of the registry (i) symptom of myocardial ischemia, (ii) new-onset significant change in ST-segment/T-wave, (iii) new-onset left bundle branch block, (iv) development of pathological Q-wave on the ECG, (v) new loss of viable myocardium or new regional wall motion abnormality on imaging studies, or (vi) intracoronary thrombus identified on coronary angiography. Patient aged 19 years or more admitted for new-onset acute MI and showing sinus rhythm (SR) will be included in this study. However, patients with (1) persistent/permanent atrial fibrillation (AF), (2) ventricular paced rhythm, (3) life-threatening co-morbidity with life expectancy $<1$ year, (4) end stage renal disease requiring renal replacement therapy, or (5) inability or unwillingness to provide consent will be excluded. The patients will be categorized into ST-segment elevation MI (STEMI) or non-STEMI by the attending physician. The extent of coronary disease will be decided by the number of major epicardial coronary arteries with significant stenosis (>50\%).

Regarding the estimation of the sample size for the present study, we decided to place no limitation on it, because there has been no registry primarily dealing with SCD in Korean patients with MI or HF. Then, it was calculated based upon the number of patients enrolled in the previous registries established in Korea ${ }^{16-19}$; 1000 consecutive patients will be enrolled for each acute MI and HF group respectively, with a total of 2,000 patients. 
All enrolled patients will provide written informed consent. Obtaining an informed consent from legal representatives will be permitted when it is impossible for the patients to provide consent by themselves due to disability. The study protocol was approved by the local institutional review board of each participating center.

\section{Study protocol and data collection}

The study is funded by a grant from the Korean Heart Rhythm Society (KHRS). The Non-Invasive Study Steering Committee of the KHRS designed the study protocol and case report forms for the present study. A web-based electronic case report form (e-CRF) will be used for prospective data collection by the attending physicians at each participating center with the assistance of a clinical research coordinator. The e-CRF for the K-REDEFINE registry was made with support from GE Healthcare Korea. However, no access to patient-related data will be allowed by the sponsor. Direct participant identifiers including names, personal identification numbers, and medical record numbers will be substituted with linking codes. Specific baseline parameters such as demographics, clinical characteristics, surface 12-lead, and ambulatory ECG data, including TWA and HRT values, echocardiographic variables, and laboratory results, will be obtained during the index admission. Table 1 shows details of the baseline clinical data that will be collected.

\section{Measurement of TWA and HRT using ambulatory ECG recording}

TWA refers to the beat-to-beat oscillation in the shape and amplitude of the ST-segment and/or T-wave (Figure 1). This alternating phenomenon has been proven to be closely related to the temporospatial heterogeneity of repolarization in many in vitro and in vivo experiments. ${ }^{12}$ In general, the greater the magnitude of TWA, the higher the risk of arrhythmogenesis. ${ }^{20-22}$ Ischemia-induced intercellular electrical decoupling and intracellular defects involving calcium handling are implicated in the development of concordant/discordant alternans followed by heterogeneous conduction block and re-entry.

HRT refers to a biphasic change in HR after ventricular premature contractions (VPCs) with initial acceleration and subsequent deceleration (Figure 2). The initial $\mathrm{HR}$ acceleration is caused by transient vagal withdrawal due to VPC-induced blood pressure (BP) lowering, whereas the subsequent $\mathrm{HR}$ deceleration is related to baroreflex-mediated transient BP elevation. Therefore, HRT assessment is an indirect assessment of baroreflex function and patients with structural heart disease such as MI and/or ischemic cardiomyopathy frequently show a blunted HRT response..$^{13,2223}$

In the present study, TWA and HRT will be prospectively measured using ambulatory ECG data in patients with acute MI. Holter electrocardiograms will be recorded during the index admission or within 3 months after discharge. An ambulatory 3-lead SEER Light Digital Holter monitor (GE Healthcare Inc., Milwaukee, WI, USA) will record ECG data at a sampling rate of 125 samples per second. All participating centers will be encouraged to record Holter ECG data for at least 20 hours to incorporate overall circadian variations in the TWA and HRT levels during daytime and nighttime. Before lead attachment, a careful skin preparation will be performed to ensure a high-quality signal and minimize noise. ${ }^{12}$ The core laboratory (Samsung Medical Center) will collect raw ambulatory ECG data recorded at each center, and analyze them with standardized analysis settings. Details are provided in Table 2. A MARS 8000 Holter analyzer (GE Healthcare Inc., Milwaukee, WI, USA) will be used for the analysis of TWA and HRT values. Three precordial leads $\left(V_{1}, V_{3}\right.$, and $\left.V_{5}\right)$ will record the electrocardiograms; the TWA value in each lead will be calculated using the modified moving average method (MMA) based on the time-domain algorithm of TWA shown in Figure 1..$^{11,13}$ Unlike the conventional spectral method that provides qualitative data only on TWA level, the MMA method provides quantitative assessment of the TWA.'2 After the initial measurement of TWA, review and confirmatory processes will be followed. During the process, 2 dedicated cardiologists with the assistance of 1 ECG specialist will examine the presence of significant noise or artifacts on the ECG recordings, and select the maximum TWA value obtained in the ECG rhythm strip without significant noise or artifacts as a final value. For HRT assessment, the following 2 quantitative values will be measured: the turbulence onset (TO) and slope (TS), as shown in Figure 2. TO is calculated using the following formula: $\mathrm{TO}=[\{(\mathrm{RR} 1+\mathrm{RR} 2)-(\mathrm{RR}-2+\mathrm{RR}-1)\} /(\mathrm{RR}-2+\mathrm{RR}-1)] \times 100$ 
Table 1. Details of baseline data in myocardial infarction study

\begin{tabular}{|c|}
\hline Demographics \\
\hline Age \\
\hline Sex \\
\hline Height/ Weight/ Body mass index \\
\hline Body surface area \\
\hline Symptom at Admission \\
\hline Chest pain \\
\hline Dyspnea with NYHA functional class \\
\hline Palpitation \\
\hline (Pre) syncope \\
\hline Medical History \\
\hline Heart failure \\
\hline Hypertension \\
\hline Diabetes \\
\hline Smoking \\
\hline Angina \\
\hline Previous PCI or $\mathrm{CABG}$ \\
\hline Previous valvular surgery \\
\hline Peripheral artery disease \\
\hline Aortic plaque \\
\hline Paroxysmal atrial fibrillation \\
\hline Stroke/TIA/systemic embolism \\
\hline $\mathrm{CHA}_{2} \mathrm{DS}_{2}$-VASc score ${ }^{*}$ \\
\hline Chronic obstructive pulmonary disease \\
\hline Chronic kidney disease \\
\hline Family history of sudden cardiac death \\
\hline Previous history of sudden cardiac arrest \\
\hline Laboratory test \\
\hline Creatine Kinase-MB \\
\hline Troponin I \\
\hline Hemoglobin \\
\hline Total/HDL/LDL cholesterol \\
\hline Blood urea nitrogen \\
\hline Creatinine \\
\hline Creatinine clearance \\
\hline Serum sodium \\
\hline Serum potassium \\
\hline High-sensitivity C-reactive protein \\
\hline N-terminal prohormone of brain natriuretic peptide \\
\hline Coronary intervention \\
\hline Chest pain onset time \\
\hline PCl time \\
\hline Infarct-related artery (left main/LAD/LCX/RCA) \\
\hline Diseases extent (1,2, or 3 vessel disease) \\
\hline Type of myocardial infarction (STEMI/non-STEMI) \\
\hline
\end{tabular}

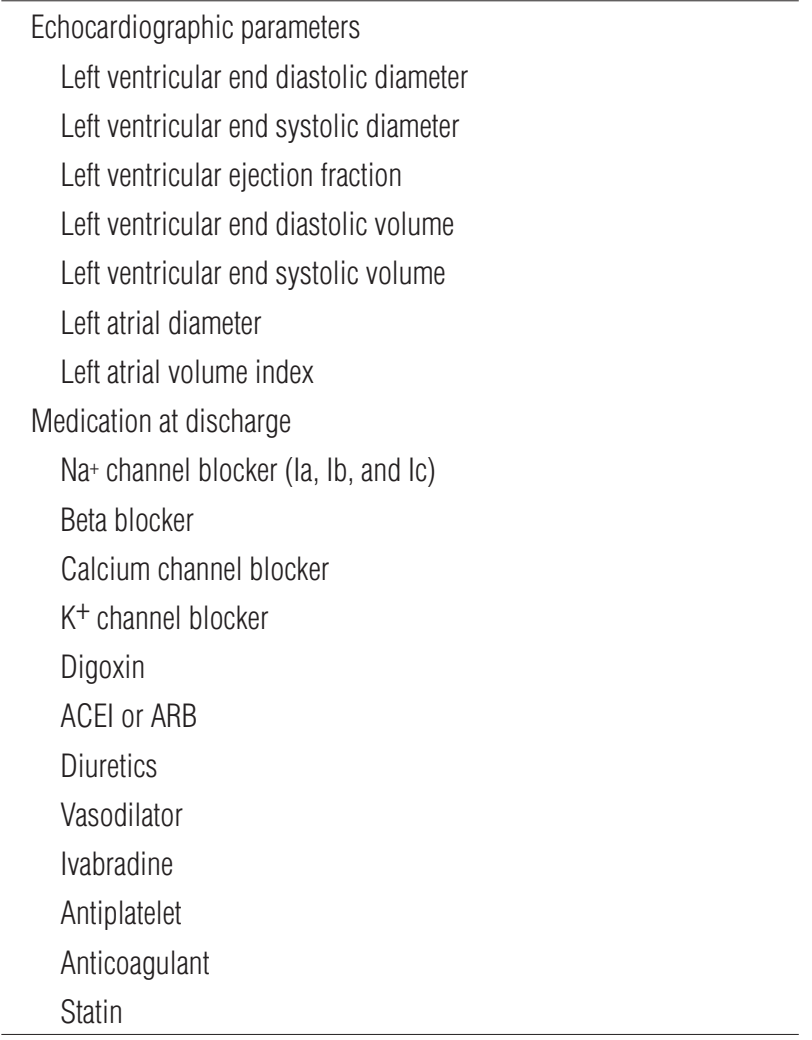

${ }^{*} \mathrm{CHA}_{2} \mathrm{DS}_{2}$-VASC score (congestive heart failure, hypertension, age $\geq 75$ years [doubled], diabetes, stroke/transient ischemic attack/thromboembolism [doubled], vascular disease [prior myocardial infarction, peripheral artery disease, or aortic plaque], age 65-75 years, sex category [female])

ACEl, angiotensin-converting enzyme inhibitor; ARB, angiotensin II receptor blocker; CABG, coronary artery bypass graft surgery; HDL, high density lipoprotein; LAD, left anterior descending coronary artery; LCX, left circumflex coronary artery; LDL, low density lipoprotein; NYHA, New York Heart Association; $\mathrm{PCI}$, percutaneous coronary intervention; RCA, right coronary carter; STEMI, ST-segment elevation myocardial infarction; TIA, transient ischemic attack

[\%], where RR-2 and RR-1 are the sinus RR intervals recorded immediately before the VPC coupling interval, and RR1 and $R R 2$ are the sinus RR intervals recorded immediately following the compensatory pause. TS is measured as the maximum positive regression slope obtained over any 5 consecutive sinus $R R$ intervals within the first 15 sinus rhythm $\mathrm{RR}$ intervals following the VPC. ${ }^{13}$ Values of TO $>0 \%$ and TS $<2.5 \mathrm{~ms} / \mathrm{RR}$ interval will be defined as abnormal, as reported in the previous studies. ${ }^{13} \mathrm{HRT}$ will be considered as abnormal when either the TO or TS values are abnormal. In addition, patients will be categorized into 3 groups as follows: HRT0 (both TO and TS normal), HRT1 

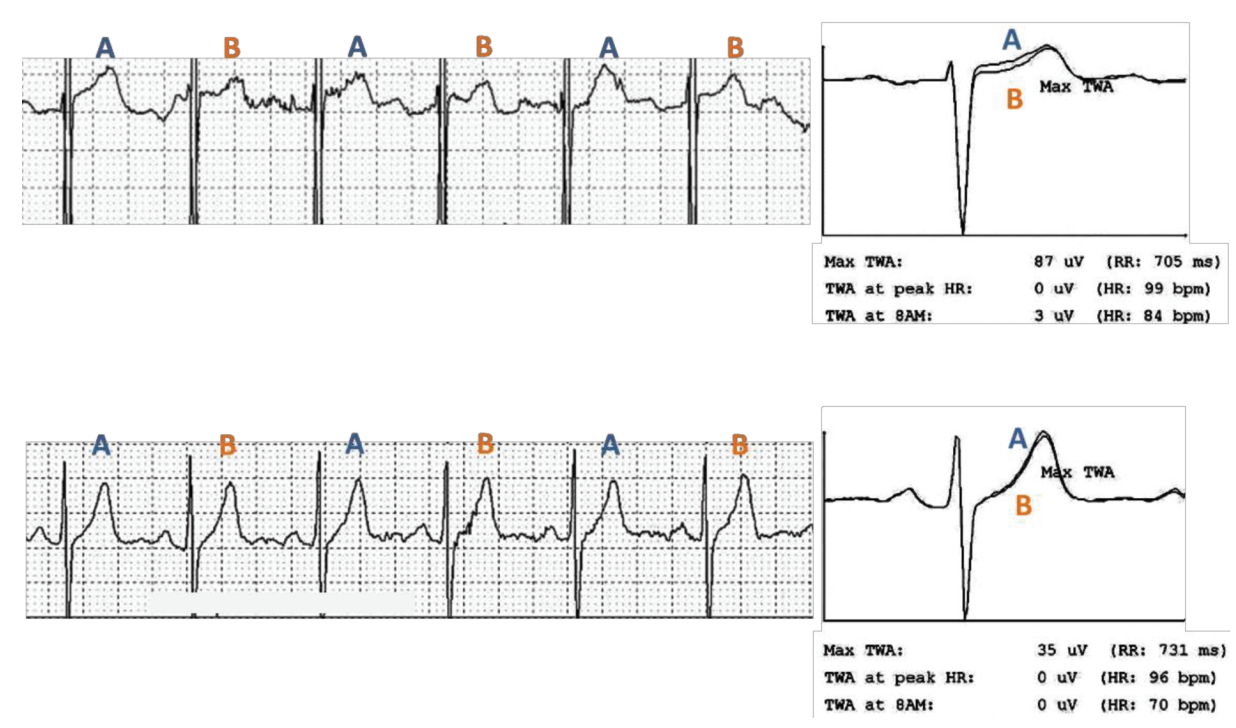

Figure 1. Measurement of T-wave alternans using moving average method bpm, beats per minute; HR, heart rate; TWA, T wave alternans

(either TO or TS abnormal), and HRT2 (both TO and TS abnormal). In addition to TWA and HRT measurements, other Holter-based variables will be collected using the same system, including time- and frequency-domain HR variability (HRV) parameters. The details of Holter-based variables analyzed in the present study are presented in Table 3.

\section{Discharge information and clinical outcomes during follow-up}

Information on the status at discharge and during follow-up will be gathered using standardized report forms. Discharge information will incorporate survival status, modes of death (noncardiac, non-sudden cardiac, or SCD) if death occurred, revascularization modalities (percutaneous intervention, bypass surgery, or medical treatment alone), types of cardiac device therapy (pacemaker, defibrillator, or biventricular pacemaker) if performed, and duration of hospitalization. Follow-up information will be collected at unplanned visits associated with study endpoints and predefined schedules, corresponding to 1,3 , 6 , and 12 months after discharge and annually thereafter up to 5 years. The primary endpoint is SCD, which is defined as unexpected death due to cardiac causes that occurs in a short time period (within 1 hour of symptom onset or unwitnessed death during sleep). Other primary and secondary outcomes are shown in Table 4. An independent Clinical Event Adjudication Committee consisting of experts in the management of patients with MI and HF will verify all clinical events such as death and re-hospitalization. The survival status and outcome data for subjects lost to follow-up will be supplemented by telephone interview in which a structured questionnaire will be used in all participating centers.

\section{Discussion}

\section{Burden of sudden cardiac death in Korea}

The annual incidence of SCD in Korea was estimated to be 40-45 per 100,000 persons according to a complete enumeration survey performed every year from 2006 by the Korean Centers for Disease Control \& Prevention using medical records of emergency medical service (EMS) including out-of-hospital SCD information; $;^{24,25}$ annually, more than 30,000 persons have experienced out-of-hospital SCD since 2009, which is similar to those from other developed countries such as the USA, Canada, Ireland, and Japan. Moreover, the annual incidence of SCD (40- 
Table 2. Details of settings for TWA and HRT measurement

Standardized Settings for TWA analysis

Update factor of $1 / 8$

Noise limit of $20 \mu \mathrm{V}$

Heart rate limit of 120 beats/min

Amplitude resolution of $5 \mu \mathrm{V}$

Characteristics of suitable VPC for HRT analysis

VPC with prematurity $>20 \%$ of the reference RR interval ${ }^{\star}$

VPC with a compensatory pause of $>120 \%$ of the reference RR interval ${ }^{*}$

VPC with 2 normal RR intervals prior to the VPC

VPC with 15 normal RR intervals after the VPC

Filter settings used to reject unsuitable rhythm strips for HRT analysis

Very short RR intervals (<200 msec.) prior to or after VPCs

Very long RR intervals (>2000 msec.) prior to or after VPCs

RR intervals with substantial difference (>200 ms) to the preceding sinus interval

RR intervals with substantial difference $(>20 \%)$ to the reference RR interval *

*Reference RR interval = mean of the 5 last sinus RR intervals preceding the VPC HRT, heart rate turbulence; VPC, ventricular premature contraction

45 per 100,000 persons) was likely underestimated, because the in-hospital SCD cases were not included in the survey. However, regrettably, the overall survival-to-discharge rates in Korea (3.0\%$4.0 \%)$ were much lower than those of other studies carried out in North America, Europe, and Japan (8\%-14\%) during the same period. 24,25

\section{Need for the K-REDEFINE registry}

A significant portion of Korean patients with MI have probably experienced SCD similar to those in other developed countries because westernized lifestyles are becoming more prevalent in Korea. Despite the clinical importance of SCD in the population with MI, previous multicenter Korean MI registries, although nationwide and well-designed, have failed to provide enough data associated with SCD; $;^{18,19}$ the Korea Acute Myocardial Infarction
Registry (KAMIR) and the Korea Working Group on Myocardial Infarction (KorMI) registries incorporating more than 34,000 patients with acute MI have provided considerable valuable insights on many aspects of acute MI. However, the KAMIR and KorMI registries did not collect data associated with SCD. Information on anti-arrhythmic medications, the use of a cardiac implantable electronic device, and ventricular arrhythmic events are also lacking.

Furthermore, this lack of data on post-MI SCD in Korea might lead to underutilization of potentially life-saving therapies such as ICD or cardiac resynchronization therapy (CRT) devices. Indeed, the rate of ICD/CRT-defibrillator (CRT-D) implantation was below $1.5 \%$ according to a recent registry study conducted in Korean patients with $\mathrm{HF}$, another high-risk group for SCD. ${ }^{26}$ Additionally, according to the 11th world survey of cardiac pacing and ICD, the annual rate of new ICD or CRT-D implantation in 


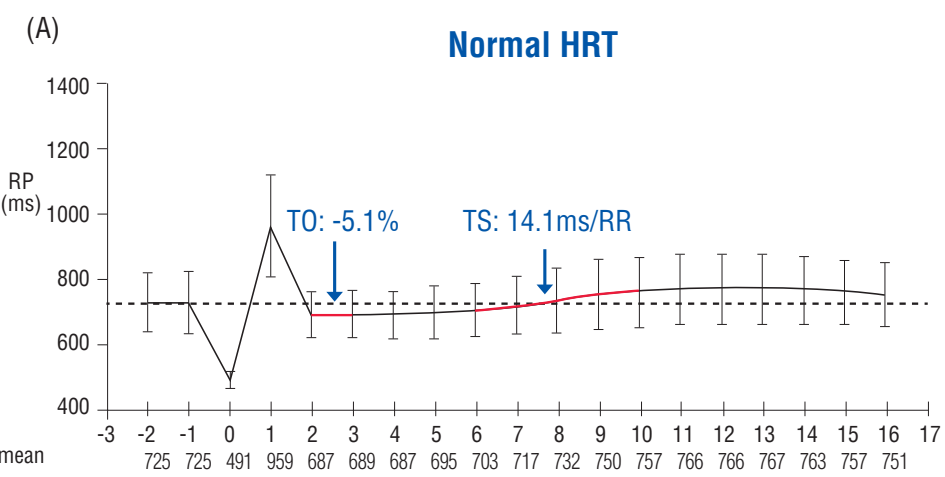

(B)

Abnormal HRT

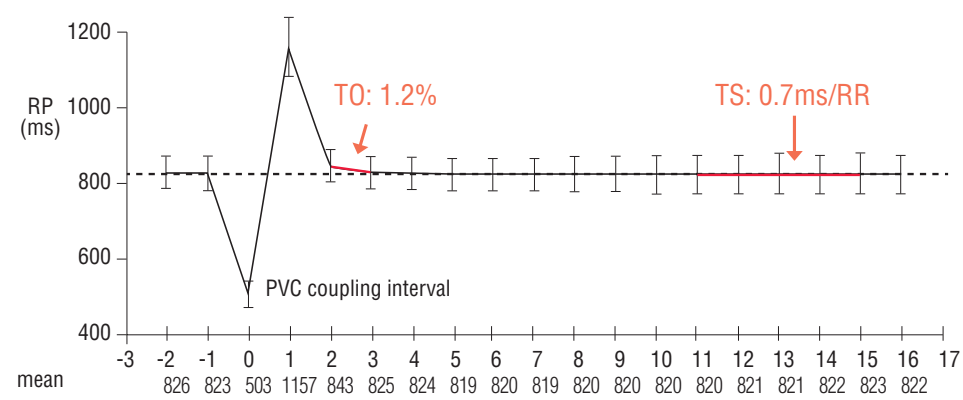

Figure 2. Representative examples of normal and abnormal heart rate turbulence $\mathrm{HRT}$, heart rate turbulence

Korea in 2009 (6 per million) was much lower than that in Japan (42 per million) or in the United States of America (434 per million), even if corrected by population. ${ }^{27}$

Given this situation, the K-REDEFINE registry will provide clinicians with valuable assistance in the identification of SCD predictors, and the adequate and timely allocation of potentially life-saving therapies to those at with MI at high risk for SCD. Indeed, another study using Korean EMS data demonstrated that witnessed collapse and a pre-hospital shockable rhythm were identified as independent predictive factors for survival-todischarge. Therefore, the adequate use of ICD/CRT-D therapy might improve outcomes in Korean patients with MI. ${ }^{28}$

\section{TWA and HRT for SCD}

Abnormal TWA and HRT, as surrogate markers of repolarization abnormality and cardiac autonomic dysfunction respectively, are closely associated with an increased risk of SCD. Therefore, they have already been investigated in many studies. However, TWA was assessed only qualitatively using a spectral method in most studies. In addition, several studies where TWA was measured quantitatively using Holter data had a limitation of retrospective analysis or small-sized nested case-control design. ${ }^{12,20,21}$ Regarding HRT, most post-MI HRT studies performed retrospective analyses and used total or cardiac death as the primary endpoint instead of SCD. ${ }^{13,22,23}$

The present K-REDEFINE study will be one of the largest cohorts to evaluate prospectively the association between TWA/ HRT and SCD in patients with MI using ambulatory ECG data. Additionally, TWA will be measured quantitatively using the MMA method. Therefore, the K-REDEFINE study could enhance our understanding of the role of TWA and HRT in identifying patients with $\mathrm{MI}$ at high risk of SCD. 
Table 3. Surface and ambulatory ECG-related variables

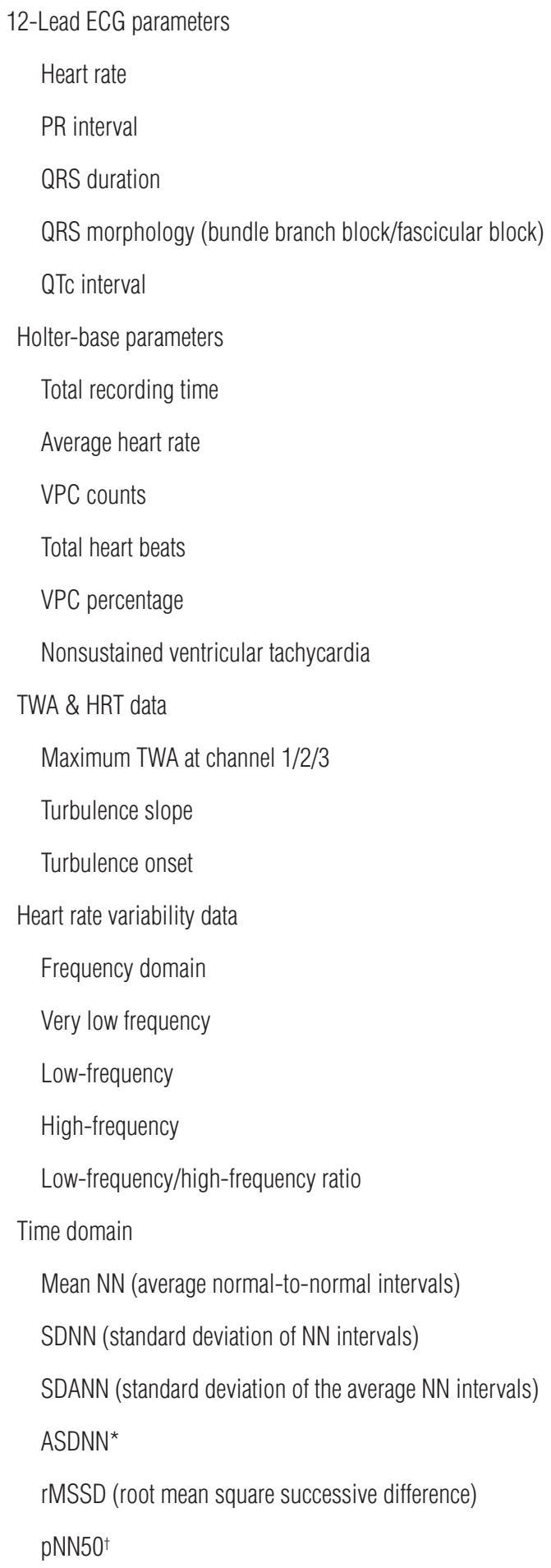

*Standard deviation of the average of NN intervals in all 5-minute segments of a 24-h recording

tPercentage of differences between adjacent NN intervals that are $>50 \mathrm{msec}$; this is one member of the larger pNNx family

ECG, electrocardiogram; HRT, heart rate turbulence; NN, normal-to-normal RR interval; VPC, ventricular premature contraction; TWA, T-wave alternans
Table 4. Study end points

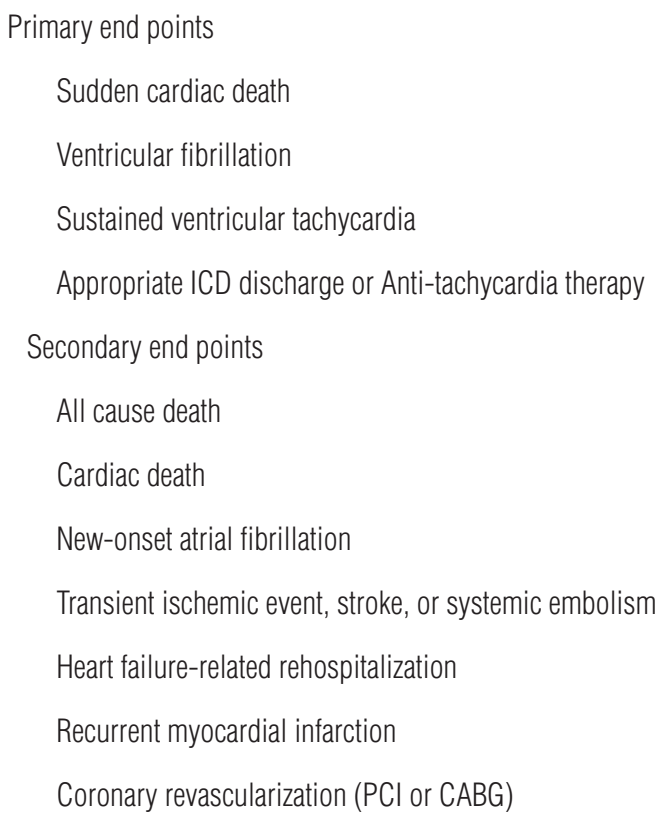

CABG, coronary artery bypass graft surgery; ICD, implantable cardioverterdefibrillator; $\mathrm{PCl}$, percutaneous coronary intervention

\section{Conclusion}

The MI study of the K-REDEFINE registry is the first Korean prospective study primarily focused on SCD in patients with MI from 25 centers in Korea. The K-REDEFINE registry will pioneer better management of patients with MI at high risk of SCD by elucidating the burden and risk factors of SCD and the clinical utility of various non-invasive ambulatory ECG-based parameters in risk stratification for SCD in this patient population.

\section{Acknowledgements}

The present study was supported by the KHRS. We express our sincere gratitude to the investigators at the 25 participating university hospitals/medical centers for their contributions to this study. The participating centers are as follows in alphabetical order: Ajou University Hospital, Asan Medical Center, Chonnam National University Hospital, Chosun University Hospital, Chung-Ang University Hospital, Chungbuk National University Hospital, Chungnam National University Hospital, Daegu 
Catholic University Medical Center, Gachon University Gil Medical Center, Inha University Hospital, Inje University Busan Paik Hospital, Inje University Ilsan Paik Hospital, International St. Mary's Hospital, Jeju National University Hospital, Keimyung University Dongsan Medical Center, Korea University Medical Center, Korea University Ansan Hospital, Kosin University Gospel Hospital, Samsung Medical Center, Sejong General Hospital, Severance Hospital of Yonsei University College of Medicine, Wonkwang University Hospital, Wonju Severance Christian Hospital, and Yeungnam University Hospital.

\section{References}

1) Adabag AS, Therneau TM, Gersh BJ, Weston SA, Roger VL. Sudden death after myocardial infarction. JAMA. 2008;300:20222029.

2) Zaman S, Kovoor P. Sudden cardiac death early after myocardial infarction: pathogenesis, risk stratification, and primary prevention. Circulation. 2014;129: 2426-2435.

3) Hong JS, Kang HC, Lee SH, Kim J.Long-term trend in the incidence of acute myocardial infarction in Korea: 1997-2007. Korean Circ J. 2009;39:467-476

4) Sim DS, Jeong MH, Kang JC.Current management of acute myocardial infarction: experience from the Korea acute myocardial infarction registry. J Cardiol. 2010; 56:1-7.

5) Moss AJ, Hall WJ, Cannom DS, Daubert JP, Higgins SL, Klein H, Levine JH, Saksena S, Waldo AL, Wilber D, Brown MW, Heo M. Improved survival with an implanted defibrillator in patients with coronary disease at high risk for ventricular arrhythmia. Multicenter automatic defibrillator implantation trial Investigators. $N$ Engl $J$ Med. 1996;335:1933-1940.

6) Buxton AE, Lee KL, Fisher JD, Josephson ME, Prystowsky EN, Hafley G.A randomized study of the prevention of sudden death in patients with coronary artery disease. Multicenter unsustained tachycardia trial investigators. N Engl J Med. 1999;341:1882-1890.

7) Moss AJ, Zareba W, Hall WJ, Klein H, Wilber DJ, Cannom DS, Daubert JP, Higgins SL, Brown MW, Andrews ML; Multicenter automatic defibrillator implantation trial II investigators. prophylactic implantation of a defibrillator in patients with myocardial infarction and reduced ejection fraction. N Engl J Med. 2002;346:877-883.
8) Gorgels AP, Gijsbers C, de Vreede-Swagemakers J, Lousberg A, Wellens HJ. Out-of-hospital cardiac arrest-the relevance of heart failure. The Maastricht circulatory arrest registry. Eur Heart J. 2003;24:1204-1209.

9) Huikuri HV, Raatikainen MJ, Moerch-Joergensen R, Hartikainen J, Virtanen V, Boland J, Anttonen O, Hoest N, Boersma LV, Platou ES, Messier MD, Bloch-Thomsen PE. Prediction of fatal or nearfatal cardiac arrhythmia events in patients with depressed left ventricular function after an acute myocardial infarction. Eur Heart J. 2009;30:689-698.

10) Hohnloser SH, Kuck KH, Dorian P, Roberts RS, Hampton JR, Hatala R, Fain E, Gent M, Connolly SJ; DINAMIT investigators. Prophylactic use of an implantable cardioverter-defibrillator after acute myocardial infarction. NEngl J Med. 2004;351:2481-2488.

11) Dagres N, Hindricks G. Risk stratification after myocardial infarction: is left ventricular ejection fraction enough to prevent sudden cardiac death? Eur Heart J. 2013;34:1964-1971.

12) Verrier RL, Klingenheben T, Malik M, El-Sherif N, Exner DV, Hohnloser SH, Ikeda T, Martínez JP, Narayan SM, Nieminen T, Rosenbaum DS. Microvolt T-wave alternans physiological basis, methods of measurement, and clinical utility--consensus guideline by International Society for Holter and Noninvasive Electrocardiology. J Am Coll Cardiol. 2011;58:1309-1324.

13) Bauer A, Malik M, Schmidt G, Barthel P, Bonnemeier H, Cygankiewicz I, Guzik P, Lombardi F, Müller A, Oto A, Schneider R, Watanabe M, Wichterle D, Zareba W. Heart rate turbulence: standards of measurement, physiological interpretation, and clinical use: International Society for Holter and Noninvasive Electrophysiology Consensus. J Am Coll Cardiol. 2008;52:1353-1365.

14) Hoshida K, Miwa Y, Miyakoshi M, Tsukada T, Yusu S, Yoshino $H$, Ikeda T. Simultaneous assessment of T-wave alternans and heart rate turbulence on holter electrocardiograms as predictors for serious cardiac events in patients after myocardial infarction. Circ J. 2013;77:432-438.

15) Slawnych MP, Nieminen T, Kähönen M, Kavanagh KM, Lehtimäki T, Ramadan D, Viik J, Aggarwal SG, Lehtinen R, Ellis L, Nikus K, Exner DV; REFINE (Risk Estimation Following Infarction Noninvasive Evaluation); FINCAVAS (Finnish Cardiovascular Study) Investigators. Post-exercise assessment of cardiac repolarization alternans in patients with coronary artery disease using the modified moving average method. J Am Coll Cardiol. 2009;53:1130-1137. 
16) Choi DJ, Han S, Jeon ES, Cho MC, Kim JJ, Yoo BS, Shin MS, Seong IW, Ahn Y, Kang SM, Kim YJ, Kim HS, Chae SC, Oh BH, Lee MM, Ryu KH; KorHF Registry. Characteristics, outcomes and predictors of long-term mortality for patients hospitalized for acute heart failure: a Report from the korean heart failure registry. Korean Circ J. 2011;41:363-371.

17) Lee SE, Cho HJ, Lee HY, Yang HM, Choi JO, Jeon ES, Kim MS, Kim JJ, Hwang KK, Chae SC, Seo SM, Baek SH, Kang SM, Oh IY, Choi DJ, Yoo BS, Ahn Y, Park HY, Cho MC, Oh BH. A multicentre cohort study of acute heart failure syndromes in Korea: rationale, design, and interim observations of the Korean acute heart failure (KorAHF) registry. Eur J Heart Fail. 2014;16:700-708

18) Song YB, Hahn JY, Kim JH, Lee SY, Choi SH, Choi JH, Choi SH, Lee SH, Yoon J, Kim YJ, Jeong MH, Gwon HC; Korea Acute Myocardial Infarction Registry Investigators. Comparison of angiographic and other findings and mortality in non-ST-segment elevation versus ST-segment elevation myocardial infarction in patients undergoing early invasive intervention. Am J Cardiol. 2010;106:1397-1403.

19) Park HW, Yoon CH, Kang SH, Choi DJ, Kim HS, Cho MC, Kim YJ, Chae SC, Yoon JH, Gwon HC, Ahn YK, Jeong MH; KAMIR/KorMI Registry. Early- and late-term clinical outcome and their predictors in patients with ST-segment elevation myocardial infarction and non-ST-segment elevation myocardial infarction. Int J Cardiol. 2013; 169:254-261.

20) Chow T, Kereiakes DJ, Bartone C, Booth T, Schloss EJ, Waller T, Chung E, Menon S, Nallamothu BK, Chan PS. Microvolt T-wave alternans identifies patients with ischemic cardiomyopathy who benefit from implantable cardioverter-defibrillator therapy. $J$ Am Coll Cardiol. 2007;49:50-58.

21) Ikeda T, Yoshino H, Sugi K, Tanno K, Shimizu H, Watanabe J,
Kasamaki Y, Yoshida A, Kato T. Predictive value of microvolt T-wave alternans for sudden cardiac death in patients with preserved cardiac function after acute myocardial infarction: results of a collaborative cohort study. J Am Coll Cardiol. 2006; $48: 2268-2274$.

22) Exner DV, Kavanagh KM, Slawnych MP, Mitchell LB, Ramadan D, Aggarwal SG, Noullett C, Van Schaik A, Mitchell RT, Shibata MA, Gulamhussein S, McMeekin J, Tymchak W, Schnell G, Gillis AM, Sheldon RS, Fick GH, Duff HJ; REFINE investigators. Noninvasive risk assessment early after a myocardial infarction the REFINE study. J Am Coll Cardiol. 2007;50:2275-2284.

23) Barthel P, Schneider R, Bauer A, Ulm K, Schmitt C, Schömig A, Schmidt G. Risk stratification after acute myocardial infarction by heart rate turbulence. Circulation. 2003;108:1221-1226.

24) Dong-Jin Oh. Overview of sudden cardiac death in Korea (out-ofhospital cardiac arrest). Int J Arrhythm. 2010;11:13-16.

25) 질병관리본부http://cdc.go.kr/CDC/contents/CdcKrContentLink. jsp? $\mathrm{fid}=30 \& \mathrm{cid}=65931 \&$ ctype $=1$

26) Park SJ, On YK, Byeon K, Kim JS, Choi JO, Choi DJ, Ryu KH, Jeon ES. Short- and long-term outcomes depending on electrical dyssynchrony markers in patients presenting with acute heart failure: clinical implication of the first-degree atrioventricular block and QRS prolongation from the Korean Heart Failure registry. Am Heart J. 2013;165:57-64.e2.

27) Mond HG, Proclemer A. The 11th world survey of cardiac pacing and implantable cardioverter-defibrillators: calendar year 2009--a World Society of Arrhythmia's project. Pacing Clin Electrophysiol. 2011;34:1013-1027.

28) Yang HJ, Kim GW, Kim H, Cho JS, Rho TH, Yoon HD, Lee MJ; NEDIS-CA Consortium. Epidemiology and outcomes in out-ofhospital cardiac arrest: a report from the NEDIS-based cardiac arrest registry in Korea. J Korean Med Sci. 2015;30:95-103. 